\title{
Regional Citrate Anticoagulation versus No-Anticoagulation for Continuous Venovenous Hemofiltration in Acute Severe Hypernatremia Patients with Increased Bleeding Risk: A Retrospective Cohort Study
}

\author{
Lijuan Zhao Feng Ma Yan Yu Yangping Li Yan Wang Lijie He Meilan Zhou \\ Xiujuan Tian Rui Jing Li Li Lu Li Chen Huang Ming Bai Shiren Sun \\ Department of Nephrology, Xijing Hospital, The Fourth Military Medical University, Xi'an, China
}

\section{Keywords}

Acute severe hypernatremia · Continuous venovenous hemofiltration - Regional citrate anticoagulation .

No-anticoagulation · Increased bleeding risk · Serum sodium reduction rate

\begin{abstract}
Purpose: This study was aimed at evaluating the efficacy and safety of regional citrate anticoagulation (RCA) versus noanticoagulation continuous venovenous hemofiltration $(\mathrm{CVVH})$ in acute severe hypernatremia patients with increased bleeding risk. Materials and Methods: Acute severe hypernatremia patients with high bleeding risk who underwent CVVH in our center between January 2011 and October 2017 were considered as candidates. Patients who were $<18$ years old, with hypovolemic hypernatremia, and had systemic anticoagulation were excluded. The included patients were divided into RCA and no-anticoagulation groups according to their anticoagulation strategy during $\mathrm{CVVH}$ and matched by age, sequential organ failure assessment scores, and vasopressor dependency. Results: Of the 64 included patients, no-anticoagulation and RCA were employed for CVVH in 23 and 41 patients, respectively. The serum sodium reduction rate (RRSeNa) was not significantly different be-
\end{abstract}

\section{KARGER}

(c) 2019 S. Karger AG, Basel

E-Mail karger@karger.com

www.karger.com/bpu tween the no-anticoagulation and RCA groups $(p=0.729)$. Compared to no-anticoagulation, RCA significantly prolonged the circuit survival time ( $15 \mathrm{~h}[4.1-23.9]$ vs. $51 \mathrm{~h}[21.3-$ 80.7], $p=0.001)$. The incidence of filter failure was $65.2 \%$ $(15 / 23)$ in the no-anticoagulation group and $2.4 \%(1 / 41)$ in the RCA group $(p<0.001)$, respectively. In the matched cohort, the RRSeNas were not different between the 2 groups $(p=0.569)$, and the filter lifespan was significantly longer in the RCA group as well $(p<0.001)$. Conclusion: RCA might be safe and effective for acute severe hypernatremia patients who underwent $\mathrm{CVVH}$ treatment. Further prospective, randomized, control trials are warranted to obtain robust evidences.

(c) 2019 S. Karger AG, Basel

\section{Introduction}

Hypernatremia is a common comorbidity in critical ill patients [1-6]. The incidences of intensive care unit acquired hypernatremia were reported to be $4 \%$ in postcardiac surgery patients [7], $24.4 \%$ in severe burn pa-

\section{L.Z., F.M., Y.Y., and Y.L. contributed equally to this work.}


tients [8], and $36.9 \%$ in severe traumatic brain injury patients [9]. Although the incidence of severe hypernatremia was only $0.6-1 \%[2,6,10]$, short-term mortality of patients with severe hypernatremia is as high as $75-87 \%$ $[11,12]$. Previous studies have reported that the reduction rate of serum sodium (RRSeNa) of conventional medical treatment was $0.11-0.36 \mathrm{mEq} / \mathrm{L} / \mathrm{h}$, and the decrease of RRSeNa was associated with an increase in patient mortality [13-17]. It is reported that continuous venovenous hemofiltration $(\mathrm{CVVH})$ could effectively reduce the serum sodium concentration in patients with acute severe hypernatremia [18-23]. Our previous studies showed that CVVH treatment most likely was more effective than conventional medical treatment for the management of acute severe hypernatremia in critically ill patients $[21,23]$. On the contrary, studies suggested that rapid-correction of hypernatremia could aggravate cerebral edema, herniation, and permanent neurologic damage, all of which were associated with increased patient mortality [15]. Although experts recommended RRSeNa of $1-2 \mathrm{mEq} / \mathrm{L} / \mathrm{h}$ for acute hypernatremia patients $[15,24]$, our previous retrospective study indicated that $\mathrm{RRSeNa}>1 \mathrm{mEq} / \mathrm{L} / \mathrm{h}$ was an independent risk factor of patient mortality in acute severe hypernatremia patients who underwent CVVH treatment $[23,25]$. These data suggested that an appropriate RRSeNa most likely could improve the outcome of acute severe hypernatremia patients.

The efficacy of CVVH is directly related to the running time of the circuit. Extracorporeal circuit clotting could decrease therapy effectiveness, and increase blood loss, circuit replacement, and costs $[26,27]$. Although standard heparin-based anticoagulation have been used to preserve the patency of extracorporeal circuit in CVVH [28], most of the severe hypernatremia patients cannot tolerate prolonged systemic anticoagulation because of the increased bleeding risk, including recent surgery or trauma, active or recent bleeding, and severe coagulopathy.

The Kidney Disease Improving Global Outcomes guideline suggested regional citrate anticoagulation (RCA) instead of heparin for CVVH in patients with increased bleeding risk and without citrate contraindication, because of the reduced bleeding incidence [29]. Additionally, our previous meta-analysis [30] demonstrated that RCA could lead to longer filter lifespan compared to the systematic and regional heparin. However, infusion of concentrated trisodium citrate (TSC) solution theoretically resulted in a significant sodium load $(408 \mathrm{mmol} / \mathrm{L}$ for $4 \%$ TSC solution). Therefore, hypernatremia was considered as one of the potential contraindications of RCA in some countries [31]. Accordingly, no-anticoagulation CVVH was commonly employed in hypernatremia patients with increased bleeding risk. However, CVVH performed without anticoagulation was usually associated with short filter lifespan (average 7-12 h) [32, 33], which reduced the therapy effectiveness and increased treatment cost. To the best of our knowledge, there was no study that assessed the safety and efficacy of RCA for $\mathrm{CVVH}$ in acute severe hypernatremia patients, and no study compared no-anticoagulation CVVH with RCA CVVH in hypernatremia patients with increased bleeding risk. Therefore, we conducted a retrospective study to evaluate the efficacy and safety of CVVH with RCA versus no-anticoagulation in acute severe hypernatremia with increased bleeding risk.

\section{Materials and Methods}

\section{Study Design and Patient Selection}

Our present study is a retrospective cohort clinical trial. Patients with acute severe hypernatremia and increased bleeding risk who received CVVH therapy in our hospital between January 2011 and October 2017 were considered for inclusion. Acute severe hypernatremia was defined as an increase in serum sodium levels from normal levels to $\geq 160 \mathrm{mEq} / \mathrm{L} / \mathrm{h}$ within $48 \mathrm{~h}[15,34]$. Increased bleeding risk was defined as the occurrence of recent (within 7 days) or active bleeding, recent trauma or surgery, recent stroke, or intracranial arteriovenous malformation or aneurysm [29]. Patients were excluded if they had any of the following conditions: (i) younger than 18 years, (ii) hypovolemic hypernatremia, and (iii) had systemic anticoagulation during the CVVH treatment.

Serum sodium levels are commonly tested on patient admission and at least every $8 \mathrm{~h}$ during $\mathrm{CVVH}$ therapy. Because of the controversial opinions on whether hypernatremia was a contraindication for RCA, the choice of anticoagulant for CVVH depended on the doctor who attended the patients in our center. And the patients were divided into RCA and no-anticoagulation groups according to their accepted anticoagulants for CVVH. Furthermore, the patients in the no-anticoagulation and RCA groups were matched at 1:1 ratio according to the following criteria: sequential organ failure assessment (SOFA; \pm 2 scores), vasopressor dependency, and age ( \pm 5 years). One of our authors (M.B.) performed matching without knowing the patient's identification characteristics and outcome data.

The study was performed in accordance with the Declaration of Helsinki. And, before CVVH treatment, all of the patients received detailed information about the advantages and disadvantages of CVVH treatment and provided a written informed consent.

\section{CVVH Treatment}

Temporary vascular access was created by inserting a dual lumen catheter into the femoral vein or jugular vein. CVVH was performed using the Prismaflex HF 100 Set system (Gambro Ho- 
spal, Stockholm, Sweden) with $2 \mathrm{~L} / \mathrm{h}$, and the blood flow was set at $200 \mathrm{~mL} / \mathrm{min}$ for no-anticoagulation CVVH and $180 \mathrm{~mL} / \mathrm{min}$ for RCA CVVH. The detailed strategies of CVVH treatment has been described in our previous studies $[21,23]$. In summary, the sodium level of the replacement fluid was initially set to be lower than the serum sodium level by $8 \mathrm{mEq} / \mathrm{L}$ and was reduced by $2 \mathrm{mEq} / \mathrm{L}$ every $4 \mathrm{~h}$. The sodium concentration of the replacement fluid was adjusted by adding $10 \%$ sodium chloride. In the RCA group, the RCA dose was modified to achieve the post-filter ionized calcium level between 0.25 and $0.35 \mathrm{mmol} / \mathrm{L}$. A serum electrolyte test $\left(\mathrm{Na}^{+}, \mathrm{K}^{+}\right.$, ionized $\mathrm{Ca}^{2+}$, and extracorporeal $\mathrm{Ca}^{2+}$ ) and blood gas analyses $\left(\mathrm{pH}, \mathrm{pCO}_{2}, \mathrm{HCO}_{3}{ }^{-}\right.$, and $\mathrm{BE}$ ) were performed every $8 \mathrm{~h}$. CVVH was continued until the correction of hypernatremia or the patient's death. Filter pressures, circuit clotting, and bleeding were monitored continuously. The filter set was scheduled for replacement every $72 \mathrm{~h}$ even without filter failure.

\section{Data Collection}

Data were retrieved from the patient medical records. Baseline characteristics, including demographic, clinical, and biochemical data at the time of diagnosis of acute severe hypernatremia were recorded. The severity of illness was scored using the acute physiology and chronic health evaluation II and the SOFA on the day of initiating CVVH. Other parameters assessed during CVVH treatment are as follows: mechanical ventilation, vasopressor dependency, laboratory parameters, fluid removal rates $(\mathrm{mL} / \mathrm{h})$, anticoagulation method, vascular access, duration of CVVH (hours), circuit coagulation, filter life (hours), filter numbers, reason for filter exchange, blood gas analyses, and serum electrolytes. The biochemical data before/after treatment and the treatment-related complications were also recorded. Two of our authors (F.M., Y.Y.) collected and entered data at the same time. The disagreements were solved by discussion and consensus.

\section{Outcomes and Definitions}

The RRSeNa was calculated as follows: RRSeNa $(\mathrm{mEq} / \mathrm{L} / \mathrm{h})=$ change of serum sodium concentration $(\mathrm{mEq} / \mathrm{L}) /$ treatment time (hours). Circuit survival duration was defined as the time from the beginning of a circle to the off-time of the filter (hemofilter clotting, persistent transmembrane pressures above $250 \mathrm{~mm} \mathrm{Hg}$, or necessitating filter replacement). The persistent TMP $>250 \mathrm{~mm} \mathrm{Hg}$ was diagnosed after the exclusion of TMP increase caused by monitor error, transient blood flow decline, and catheter dysfunction. The changes in hemoglobin $(\triangle \mathrm{HB}) /$ platelet count $(\triangle \mathrm{PLT})$ were calculated as follows: $\Delta \mathrm{HB}(\mathrm{g} / \mathrm{L}) / \Delta \mathrm{PLT}\left(10^{9} / \mathrm{L}\right)=\mathrm{HB}$ concentration/ PLT count after treatment - HB concentration/PLT count before treatment. Citrate accumulation was identified if the patient fulfilled 2 or more of the following criteria: a ratio of total calcium to ionized calcium $>2.5$, clinical signs of hypocalcemia (titanic symptoms or prolonged QT interval not due to medication), or progressive acidosis $(\mathrm{pH}<7.20)$ with an increased anion gap $(>13 \mathrm{mmol} / \mathrm{L})$ in the presumed absence of anions other than citrate. The filterrelated outcomes were observed during the CVVH treatment, and the patient survival was observed during their hospital stay. No patients were lost during our observational period.

\section{Statistical Analysis}

Statistical analysis was performed using the SPSS 16.0 software package. Continuous variables were presented as means \pm $\mathrm{SD}$, and categorical variables were presented as event number and percentage (\%). Continuous variables that were normally distributed were compared using a $t$ test; otherwise, a MannWhitney rank test was employed. We used $\chi^{2}$ test or Fisher's exact test for categorical variables. Circuit survival time was calculated using Kaplan-Meier curves and compared using log-rank test. Independent predictors of filter span were evaluated using the Cox regression model. The covariates included in univariate analysis were age, sex, comorbidity, temporary vascular access, baseline PLT count, HB, prothrombin time (PT), activated partial thromboplastin time (APTT), serum sodium, serum creatinine, blood urea nitrogen, serum albumin, serum total bilirubin, neurologic disease, and the anticoagulation strategy. Variables with $p<0.05$ in univariate analysis and clinical important variables which were not identified as a risk factor in univariate analysis, including PLT counts and vascular access site, were incorporated into the multivariate Cox regression analysis. Before the performance of the multivariate Cox regression analysis, collinearity diagnosis and the relation analysis were conducted and only one of the variables with significant correlation was included in the multivariate analysis. The data of the patient baseline characteristics and major endpoints were available in all of the included patients. Mean imputation was employed in the condition of missing data. A two-tailed $p<0.05$ was considered statistical significant.

\section{Results}

\section{Baseline Characteristics}

A total of 101 acute severe hypernatremia patients with increased bleeding risk received CVVH between March 2011 and October 2017 in our center. Of which, 37 patients were excluded because of younger than 18 years $(n=5)$, hypovolemic hypernatremia $(n=7)$, and systemic anticoagulation $(n=25)$. Of the 64 included patients, no-anticoagulation was used in 23 patients and RCA was used in 41 patients (Fig. 1).

The baseline characteristics of the included patients are presented in Table 1 . The coagulation status including PLT count, PT, and APTT were not significantly different between the 2 groups. The 2 groups were significantly different in age $(p=0.049)$, SOFA score $(p=0.003)$, the percentage of neurologic disease $(p=0.044)$.

\section{Correction of Hypernatremia}

The serum sodium concentration before and after CVVH treatment were available in all of the included patients. The mean serum sodium concentration before CVVH treatment was $174.7 \pm 8.7$ and $172.5 \pm 9.2 \mathrm{mEq} / \mathrm{L}$ in no-anticoagulation and RCA groups, respectively $(p=0.352)$. The average CVVH time was $29.2 \pm 18.2 \mathrm{~h}$ in the no-anticoagulation group and $27.8 \pm 15.7 \mathrm{~h}$ in the RCA group ( $p=0.753)$. The mean serum sodium con-
46

Blood Purif 2020;49:44-54 DOI: $10.1159 / 000502937$
Zhao/Ma/Yu/Li/Wang/He/Zhou/Tian/ Jing/Li/Li/Huang/Bai/Sun 
Table 1. Baseline characteristics of the included patients in the original cohort

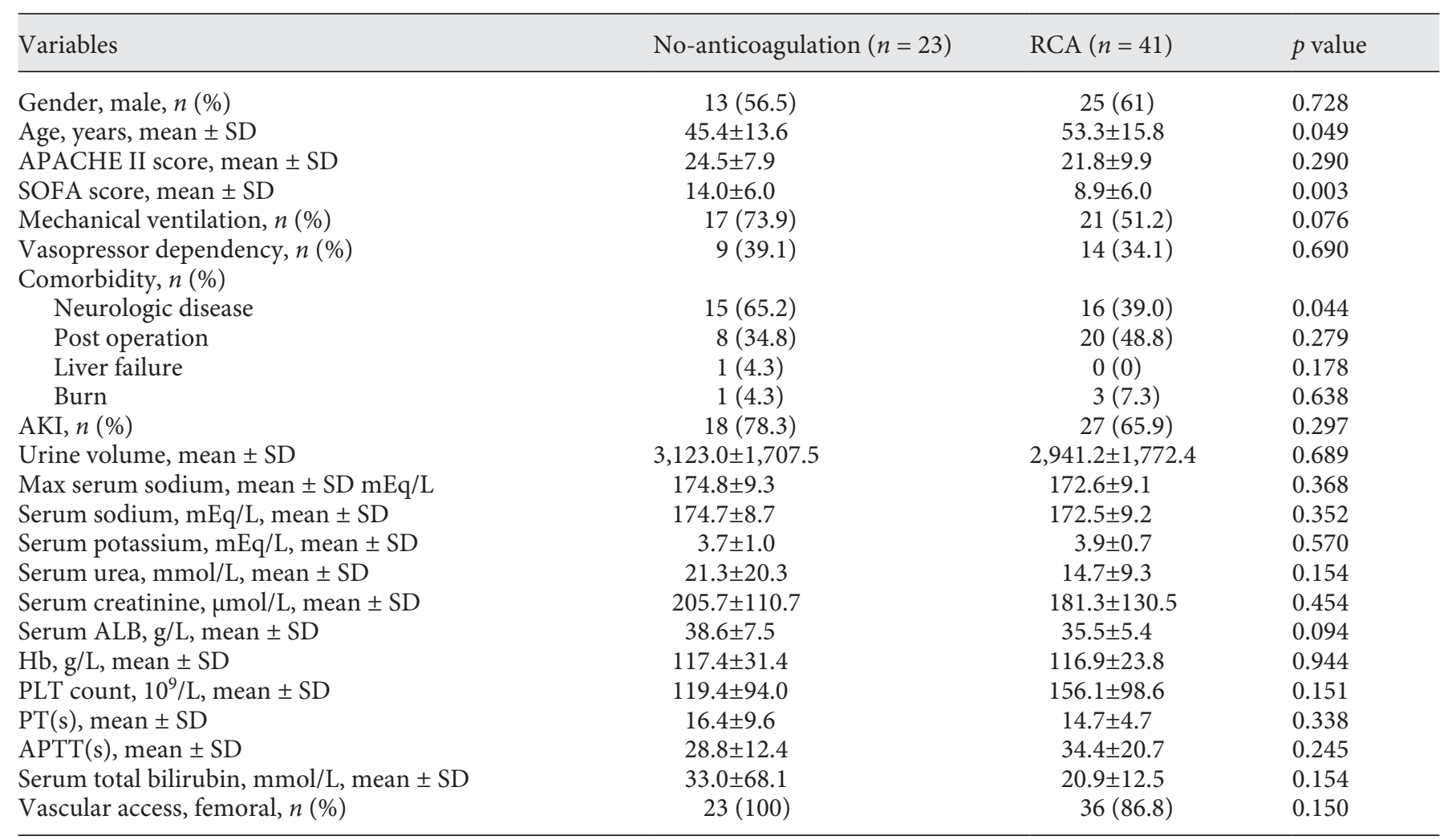

RCA, regional citrate anticoagulation; APACHE II, the Acute Physiology and Chronic Health Evaluation II; SOFA, sequential organ failure assessment; HB, hemoglobin; PLT, platelet; PT, prothrombin time; APTT, activated partial thromboplastin time.

Table 2. The outcomes of mean serum sodium reduction rate, filter, and patient mortality in the original cohort

\begin{tabular}{|c|c|c|c|}
\hline Variables & $\begin{array}{l}\text { No-anticoagulation } \\
(n=23)\end{array}$ & $\begin{array}{l}\mathrm{RCA} \\
(n=41)\end{array}$ & $p$ value \\
\hline Mean hypernatremia correction rates, $\mathrm{mEq} / \mathrm{L} / \mathrm{h}$ & $0.95 \pm 0.39$ & $0.92 \pm 0.28$ & 0.729 \\
\hline Estimated median survival time of the first circuit (95\% CI ) & $14.0(4.1-23.9)$ & $51.0(21.3-80.7)$ & $<0.001$ \\
\hline Estimated median survival time of all circuits $(95 \% \mathrm{CI})$ & $16.0(11.8-20.2)$ & $51.0(19.3-82.7)$ & $<0.001$ \\
\hline Number of filters, median (range) & $2(1-4)$ & $1(1-2)$ & $<0.001$ \\
\hline Filter failure percent, $n(\%)$ & $65.2(15 / 23)$ & $2.4(1 / 41)$ & $<0.001$ \\
\hline Mortality rate, $n(\%)$ & $19(82.6)$ & $25(61.0)$ & 0.073 \\
\hline
\end{tabular}

RCA, regional citrate anticoagulation.

centration after CVVH treatment was $151.9 \pm 9.8$ and $148.4 \pm 7.9 \mathrm{mEq} / \mathrm{L}$ in the no-anticoagulation and RCA groups, respectively $(p=0.117)$. The mean RRSeNa was $0.95 \pm 0.39 \mathrm{mEq} / \mathrm{L} / \mathrm{h}$ in the no-anticoagulation group and $0.92 \pm 0.28 \mathrm{mEq} / \mathrm{L} / \mathrm{h}$ in the RCA group $(p=0.729$, Table 2).

RCA-CVVH for Acute Severe

Hypernatremia

\section{Circuit Survival Time}

The filter survival time was available in all of the included patients. The estimated median survival time of the first circuit was $14.0 \mathrm{~h}(95 \% \mathrm{CI} 4.1-23.9)$ in the noanticoagulation group and $51.0 \mathrm{~h}$ (95\% CI 21.3-80.7) in the RCA group. The estimated median survival time of all 
Fig. 1. Inclusion flowchart. CVVH, continuous venovenous hemofiltration; RCA, regional citrate anticoagulation.

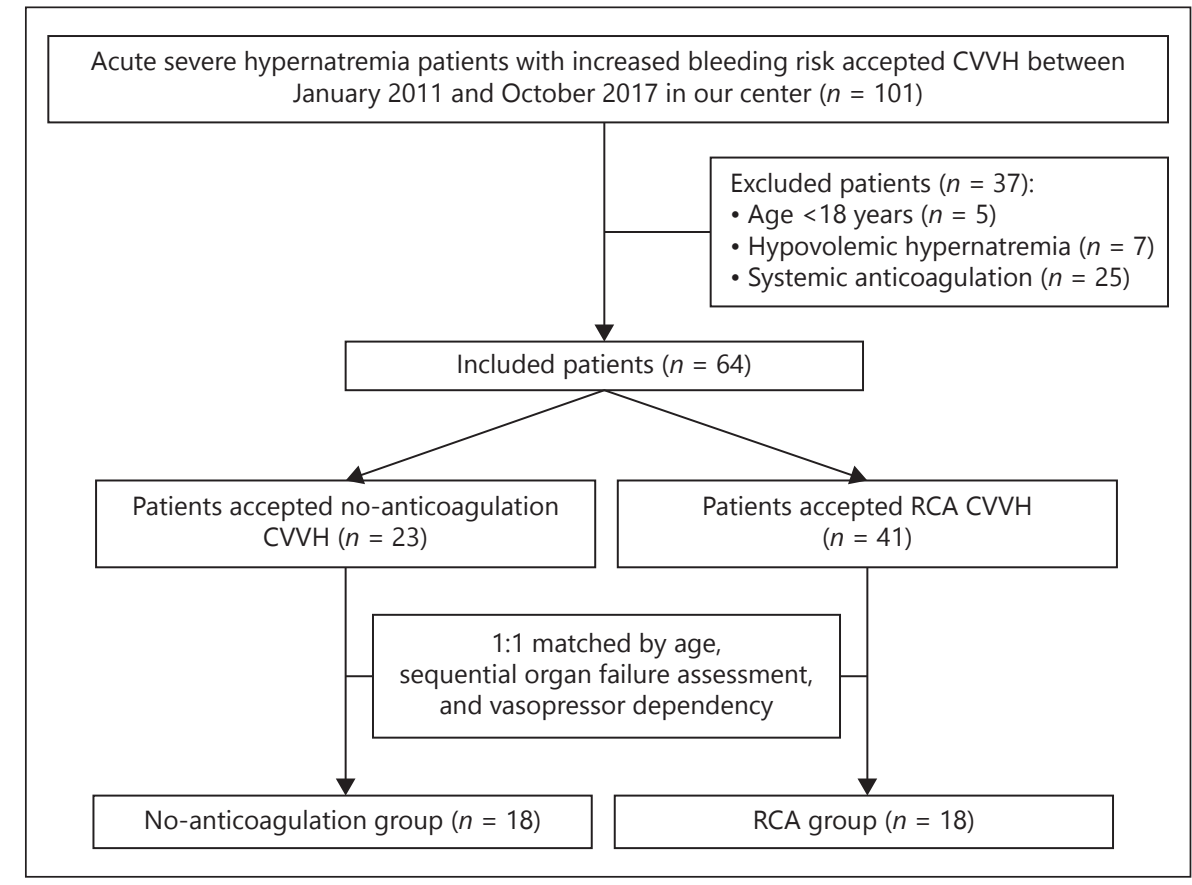

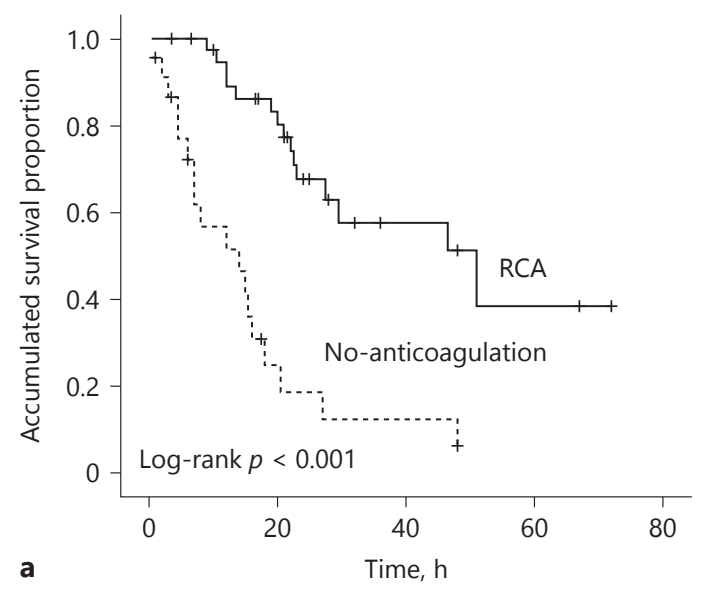

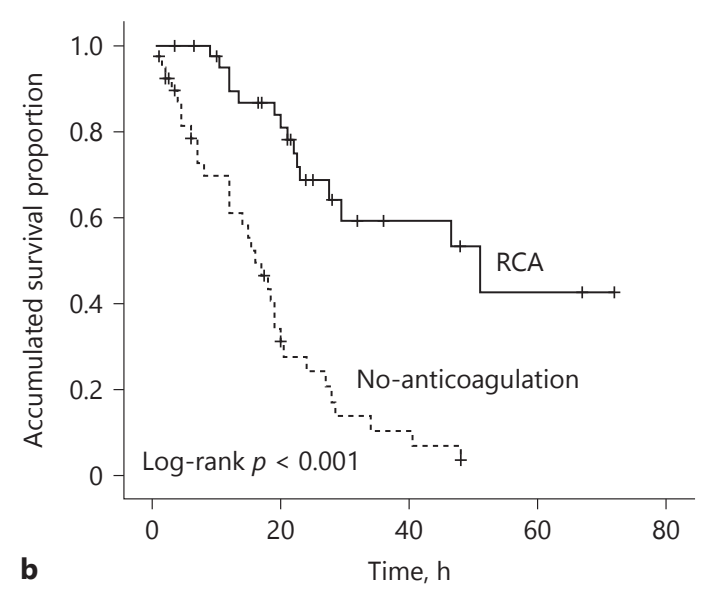

Fig. 2. Kaplan-Meier survival curves show the accumulated survival time of the first filter (a) and all filters (b) in the original cohort. RCA, regional citrate anticoagulation.

circuits was $16.0 \mathrm{~h}(95 \% \mathrm{CI} 11.8-20.2)$ in the no-anticoagulation group and $51.0 \mathrm{~h}(95 \% \mathrm{CI} 19.3-82.7)$ in the RCA group. The survival time of the first circuit and all circuits were significantly longer in the RCA group compared to the no-anticoagulation group in the original cohort $(p<0.001$, Fig. 2a, b). The incidence of filter failure was $65.2 \%(15 / 23)$ in the no-anticoagulation group and $2.4 \%(1 / 41)$ in the RCA group $(p<0.001)$. The median numbers of used filters were 2 (range 1-4) and 1 (range
$1-2)$ in the no-anticoagulation group and the RCA group, respectively $(p<0.001$, Table 2$)$.

In the univariate analysis, RCA $(p<0.001)$, baseline PT $(p=0.035)$, APTT $(p=0.002)$, serum albumin $(p=$ $0.037)$, and neurologic disease $(p=0.019)$ were significantly related to the first filter survival time. The correlation analysis demonstrated that PT and APTT were significantly relevant $(r=0.669, p=0.000)$. Therefore, only one of these 2 variables was included in the Cox regres-
48

Blood Purif 2020;49:44-54

DOI: $10.1159 / 000502937$
Zhao/Ma/Yu/Li/Wang/He/Zhou/Tian/ Jing/Li/Li/Huang/Bai/Sun 
Table 3. Univariate and multivariable Cox regression analyses of the risk factors of the first filter survival time in the original cohort

\begin{tabular}{|c|c|c|c|c|c|c|c|c|c|c|c|c|c|c|c|}
\hline \multirow[t]{2}{*}{ Variables } & \multicolumn{3}{|c|}{ Univariate analysis } & \multicolumn{3}{|c|}{$\begin{array}{l}\text { Multivariate analysis } \\
\left(\text { model } 1^{\mathrm{a}}\right)\end{array}$} & \multicolumn{3}{|c|}{$\begin{array}{l}\text { Multivariate analysis } \\
\left(\operatorname{model} 2^{\mathrm{b}}\right)\end{array}$} & \multicolumn{3}{|c|}{$\begin{array}{l}\text { Multivariate analysis } \\
\left(\text { model } 3^{c}\right)\end{array}$} & \multicolumn{3}{|c|}{$\begin{array}{l}\text { Multivariate analysis } \\
\left(\text { model } 4^{\mathrm{d}}\right)\end{array}$} \\
\hline & HR & $95 \% \mathrm{CI}$ & $p$ value & HR & $95 \% \mathrm{CI}$ & $p$ value & HR & $95 \% \mathrm{CI}$ & $p$ value & HR & $95 \% \mathrm{CI}$ & $p$ value & HR & $95 \% \mathrm{CI}$ & $p$ value \\
\hline RCA (yes/no) & 0.02 & $0.003-0.155$ & $<0.001$ & 0.033 & $0.004-0.279$ & 0.002 & 0.013 & $0.001-0.125$ & $<0.001$ & 0.020 & $0.002-0.258$ & 0.003 & 0.007 & $0.001-0.106$ & $<0.001$ \\
\hline Baseline APTT & 0.835 & $0.746-0.935$ & 0.002 & 0.874 & $0.793-0.964$ & 0.007 & & & & & & & & & \\
\hline Baseline PT & 0.766 & $0.598-0.981$ & 0.035 & & & & 0.642 & $0.481-0.857$ & 0.003 & & & & 0.703 & $0.524-0.943$ & 0.019 \\
\hline Baseline serum Alb & 1.09 & $1.005-1.181$ & 0.037 & 1.07 & $1.001-1.143$ & 0.046 & & & & & & & & & \\
\hline \multicolumn{16}{|c|}{ Neurologic disease } \\
\hline (yes/no) & 3.915 & $1.256-12.20$ & 0.019 & & & & & & & & & & & & \\
\hline Baseline PLT & 1.001 & $0.996-1.006$ & 0.632 & & & & & & & & & & & & \\
\hline Vascular access site & 0.042 & $0.000-45.473$ & 0.373 & & & & & & & & & & & & \\
\hline
\end{tabular}

a Model 1 was adjusted for RCA, baseline APTT, Alb, neurologic disease.

${ }^{\mathrm{b}}$ Model 2 was adjusted for RCA, baseline PT, Alb, neurologic disease.

${ }^{c}$ Model 3 was adjusted for RCA, baseline APTT, Alb, neurologic disease, baseline PLT, vascular access site.

${ }^{\mathrm{d}}$ Model 4 was adjusted for RCA, baseline PT, Alb, neurologic disease, baseline PLT, vascular access site.

RCA, regional citrate anticoagulation; APTT, activated partial thromboplastin time; PLT, platelet; PT, prothrombin time; Alb, albumin; HR, hazard ratio.

sion model 1 and 2 to avoid the influence of parameter collinearity. Model 1 showed that RCA (HR 0.033, 95\% CI 0.004-0.279, $p=0.002$ ), baseline APTT (HR 0.874, $95 \%$ CI $0.793-0.964, p=0.007$ ), and baseline serum albumin (HR 1.07, 95\% CI 1.001-1.143, $p=0.046$ ) were independent risk factors of the filter survival time. Model 2 showed that RCA (HR 0.013, 95\% CI 0.001-0.125, $p<0.001)$ and baseline PT (HR 0.642, 95\% CI 0.481$0.857, p=0.003$ ) were independent risk factors of the filter survival time. Model 3 showed that only RCA (HR $0.020,95 \%$ CI $0.002-0.258, p=0.003$ ) was the independent risk factor of the filter survival time. Model 4 showed that RCA (HR 0.007, 95\% CI 0.001-0.106, $p<0.001$ ) and baseline PT (HR 0.703, 95\% CI 0.524-0.943, $p=0.019$ ) were independent risk factors of the filter survival time. In all of the 4 Cox regression models, RCA was identified as one of the independent risk factors of the filter survival time (Table 3 ).

\section{HB Concentration and Coagulation Indicators}

The value of HB and PLT count after CVVH was not available in the treatment of 8 patients $(8 / 64,12.5 \%)$. The mean $\Delta \mathrm{HB}$ was $-13.8 \pm 20.0 \mathrm{~g} / \mathrm{L}$ in the no-anticoagulation group and $-4.7 \pm 24.2 \mathrm{~g} / \mathrm{L}$ in the RCA group $(p=$ $0.159)$. And, the mean $\triangle$ PLT were $-64.6 \pm 61.8 \times 10^{9} / \mathrm{L}$ and $-38.2 \pm 66.1 \times 10^{9} / \mathrm{L}$ in the no-anticoagulation and RCA groups, respectively ( $p=0.149)$. The PLT count after treatment had significant difference between the 2 groups ( $p=0.002$, Table 4$)$. In the no-anticoagulation group, the PLT count significantly decreased after CVVH treatment $(p=0.026)$. The data of coagulation indicators after CVVH treatment was not available in 21 patients $(21 / 64$, $32.8 \%)$. In both groups, PT did not significantly change after CVVH treatment $(p=0.533, p=0.647)$ and APTT also did not significantly change after CVVH treatment $(p=0.165, p=0.342)$.

\section{Adverse Events}

Three patients $(3 / 23,13.0 \%)$ in the no-anticoagulation group developed a bleeding episode compared to 1 patient $(1 / 41,2.4 \%)$ in the RCA group $(p=0.128)$. Only 1 patient $(1 / 41,2.4 \%)$ in the RCA group switched to no-anticoagulation because of alkalosis after $10 \mathrm{~h}$ RCA CVVH therapy. The mean total calcium was $2.21 \pm 0.19 \mathrm{mmol} / \mathrm{L}$ in the noanticoagulation group and $2.15 \pm 0.27 \mathrm{mmol} / \mathrm{L}$ in the RCA group $(p=0.667)$. In the RCA group, the mean ionized calcium was $1.01 \pm 0.14 \mathrm{mmol} / \mathrm{L}$, and the mean totCa/ ionCa ratio was $2.13 \pm 0.20$. TotCa/ionCa $>2.5$ was not observed in all the patients. None of the patients had clinical symptoms of citrate accumulation.

\section{Mortality}

The average follow-up time for patient survival was $15.0 \pm 19.9$ days. The in-hospital mortality rates were $82.6 \%(19 / 23)$ and $61.0 \%(25 / 41)$ in the no-anticoagulation and RCA groups, respectively $(p=0.073)$.

\section{Outcomes of the Matched Cohort}

According to the matching criteria, 18 patients in the no-anticoagulation $\mathrm{CVVH}$ treatment group and 18 patients in the RCA CVVH group were matched. The 2 matched groups did not differ significantly in the baseline characteristics (online suppl. Table 1; for all online suppl. material, see www.karger.com/doi/10.1159/000502937). The mean RRSeNas were $0.94 \pm 0.41$ and $0.86 \pm 0.36$ $\mathrm{mEq} / \mathrm{L} / \mathrm{h}$ in the no-anticoagulation and RCA groups, re- 

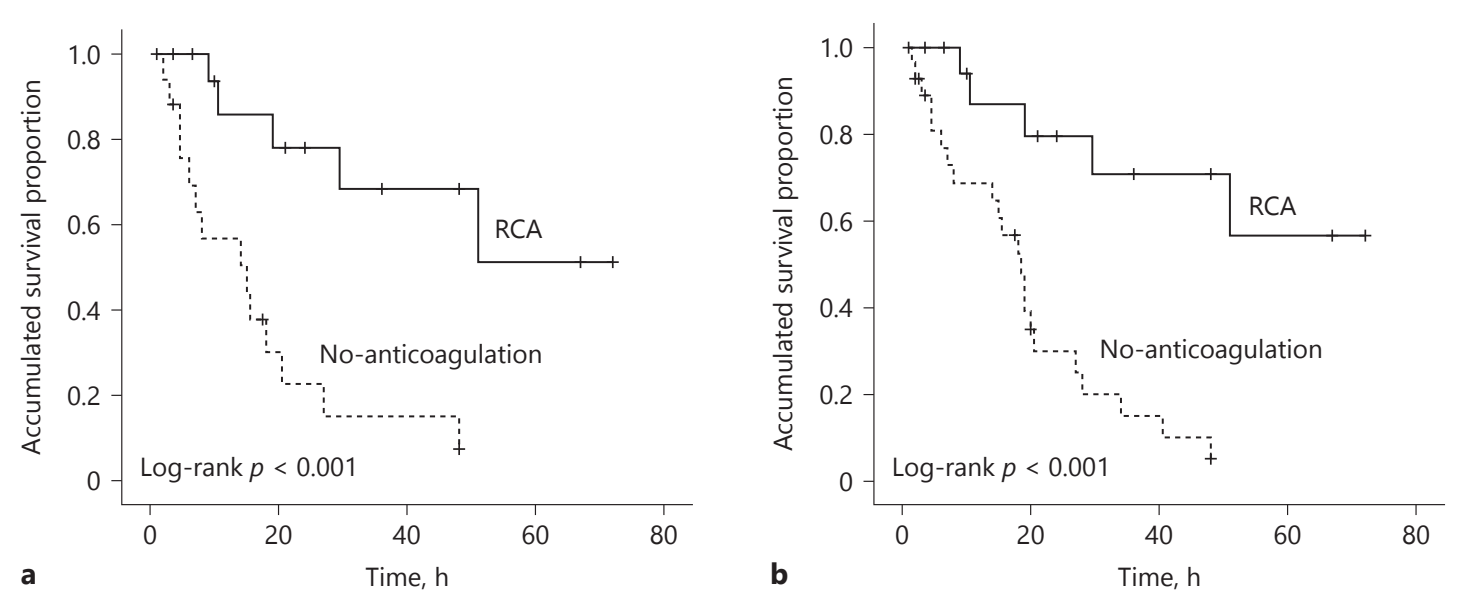

Fig. 3. Kaplan-Meier survival curves show the accumulated survival time of the first filter (a) and all filters (b) in the matched cohort. RCA, regional citrate anticoagulation.

Table 4. $\mathrm{Hb}$ concentration and coagulation indicators at the baseline and after CVVH treatment in the original cohort

\begin{tabular}{|c|c|c|c|}
\hline Variables & No-anticoagulation $(n=23)$ & $\operatorname{RCA}(n=41)$ & $p$ value \\
\hline \multicolumn{4}{|l|}{$\mathrm{Hb}, \mathrm{g} / \mathrm{L}$, mean $\pm \mathrm{SD}$} \\
\hline Before treatment & $117.4 \pm 31.4$ & $116.9 \pm 23.8$ & 0.944 \\
\hline After treatment & $106.7 \pm 27.5$ & $111.8 \pm 23.8$ & 0.471 \\
\hline$\Delta \mathrm{Hb}$ & $-13.8 \pm 20.0$ & $-4.7 \pm 24.2$ & 0.159 \\
\hline$p$ value & 0.247 & 0.356 & \\
\hline \multicolumn{4}{|c|}{ PLT count, $10^{9} / \mathrm{L}$, mean $\pm \mathrm{SD}$} \\
\hline Before treatment & $119.4 \pm 94.0$ & $156.1 \pm 98.6$ & 0.151 \\
\hline After treatment & $67.6 \pm 46.1$ & $124.9 \pm 87.7$ & 0.002 \\
\hline$\Delta \mathrm{PLT}$ & $-64.6 \pm 61.8$ & $-38.2 \pm 66.1$ & 0.149 \\
\hline$p$ value & 0.026 & 0.149 & \\
\hline \multicolumn{4}{|l|}{$\mathrm{PT}(\mathrm{s})$, mean $\pm \mathrm{SD}$} \\
\hline Before treatment & $16.4 \pm 9.6$ & $14.7 \pm 4.7$ & 0.338 \\
\hline After treatment & $14.9 \pm 2.5$ & $15.2 \pm 4.1$ & 0.807 \\
\hline$\Delta \mathrm{PT}$ & $-0.9 \pm 2.6$ & $0.3 \pm 2.5$ & 0.135 \\
\hline$p$ value & 0.533 & 0.674 & \\
\hline \multicolumn{4}{|l|}{$\operatorname{APTT}(\mathrm{s})$, mean $\pm \mathrm{SD}$} \\
\hline Before treatment & $28.8 \pm 12.4$ & $34.4 \pm 20.7$ & 0.245 \\
\hline After treatment & $41.0 \pm 38.5$ & $38.6 \pm 11.8$ & 0.771 \\
\hline$\triangle \mathrm{APTT}$ & $14.9 \pm 36.8$ & $3.2 \pm 23.6$ & 0.210 \\
\hline$p$ value & 0.165 & 0.342 & \\
\hline
\end{tabular}

HB, hemoglobin; CVVH, continuous venovenous hemofiltration; RCA, regional citrate anticoagulation; PLT, platelet; PT, prothrombin time; APTT, activated partial thromboplastin time.

spectively, ( $p=0.569$, online suppl. Table 2$)$. The estimated median survival time of the first circuit was $15.0 \mathrm{~h}$ (95\% CI 1.5-28.5) in the no-anticoagulation group and $>72 \mathrm{~h}$ in the RCA group $(p<0.001)$. And the estimated median survival time of all circuits was $18.5 \mathrm{~h}(95 \% \mathrm{CI}$
14.5-22.5) in the no-anticoagulation group and $>72 \mathrm{~h}$ in the RCA group (Fig. 3a, b, $p<0.001$ ). The PLT count significantly decreased after CVVH treatment in the no-anticoagulation group ( $p=0.035$; online suppl. Table 3 ). Three patients $(13.0 \%)$ in the no-anticoagulation group 
and no patient in the RCA group developed a bleeding episode. The mortality rates of the 2 matched groups were both $83.3 \%(15 / 18)$.

\section{Discussion}

There are controversial opinions on the choice of anticoagulation during CVVH therapy in acute severe hypernatremia patients with increased bleeding risk. The results of our present study suggest that: (1) RCA CVVH could reduce the serum sodium as effective as no-anticoagulation CVVH and (2) RCA significantly prolonged the filter survival time and decreased the number of used filters compared with no-anticoagulation. In addition, no obvious citrate accumulation was observed in the RCA CVVH group.

Hypernatremia was considered to be one of the major side effects of RCA [35]. In clinical practice, $4 \%$ TSC was commonly used for RCA CVVH, which has $408 \mathrm{mmol} / \mathrm{L}$ sodium and could induce a significant sodium load leading to hypernatremia. Klingele et al. [36] used a dialysate solution with a reduced concentration of sodium to avoid hypernatremia in severe liver dysfunction patients who accepted RCA CVVHD. In our study, compared to the no-anticoagulation group, the RCA group had a similar RRSeNa without additional sodium level reduction of the replacement solution. Our previous study [25] suggested that the RRSeNa most likely should be controlled between 0.5 and $1.0 \mathrm{mEq} / \mathrm{L} / \mathrm{h}$ to reduce the mortality risk. In the original cohort of our present study, the RRSeNa was $0.95 \mathrm{mEq} / \mathrm{L} / \mathrm{h}$ in the no-anticoagulation group and $0.92 \mathrm{mEq} / \mathrm{L} / \mathrm{h}$ in the RCA group, both of which were within the suggested range. In the matched group, the RRSeNa of both groups were within $0.5-1.0 \mathrm{mEq} / \mathrm{L} / \mathrm{h}$. Accordingly, CVVH with either no-anticoagulation or RCA could effectively correct the serum sodium level with targeted RRSeNa.

One of the most frequent problems encountered in patients treated by CVVH was indeed circuit downtime, implying that the delivered RRT dose was often lower than the prescribed dose [37]. Randomized studies report similar or longer circuit survival and less bleeding with citrate compared to heparin [37-41]. Our previous meta-analysis [30] also demonstrated that RCA could lead to a longer filter survival time compared to systematic heparin. CVVH with RCA was also proved superior to anticoagulant-free CVVH in critically ill patients, for whom systemic anticoagulation was contraindicated [33, 42]. Our results demonstrated that the median filter survival time of the RCA group was 3 times longer than the no-anticoagulation group. The prolonged survival time reduced the treatment interruption and downtime, which has been considered a key quality indicator for CVVH performance [43]. Additionally, the prolonged filter lifespan also decreased the incidence of filter clotting. In our original cohort, filter clotting occurred in $2.4 \%$ of the patients in the RCA group versus $65.2 \%$ of the patients in the no-anticoagulation group. Increased filter clotting could increase the no-bleeding blood lost. In the no-anticoagulation group, the concentrations of HB and PLT demonstrated an obvious downward trend. However, limited by our sample size, no significant difference between the 2 groups was observed. Furthermore, the prolonged lifespan could reduce the number of used filters and the cost of CVVH treatment. From the start of CVVH to the correction of hypernatremia, we observed that the no-anticoagulation patients used significantly more filers than the RCA patients. Oudemans-van Straaten [44] suggested that RCA could ensure enough filter life and decrease treatment cost compared with heparin anticoagulation for CVVH.

Metabolic abnormalities such as alkalosis, acidosis, and electrolyte abnormalities can result from RCA due to the buffering capacity of citrate, and the loss of calcium bound to citrate in the effluent [45]. Metabolic complications could be reduced if a standardized protocol is applied to adjust dialysate flow and calcium substitution so that the blood PH and ionCa levels would be maintained within a normal range [46]. In our study, we monitored blood gas and electrolyte every $8 \mathrm{~h}$ at least and adjusted prescription parameters according to the results in time. We found that only 1 patient in the RCA group had alkalosis, which was quickly corrected after the stop of RCA. The incidences of ionCa disorder were not significantly different between the 2 groups.

In the original cohort, the mortality of acute severe hypernatremia was $68.8 \%$, which had a downward trend in the RCA groups although the difference is not significant. After the 2 groups were matched using SOFA, age, and vasopressor dependency, the mortality of the 2 groups was both $83.3 \%$. The mortality was in concordance with previous reports from other center, but higher than our previous study (58.1\%) [25]. This heterogeneity most likely attributed to the included patients of our present study were more severe regarding the higher SOFA score and increased bleeding risk.

Compared with previous studies, we think that there are several strengths of our present study. At first, to the best of our knowledge, this is the first study about the efficacy and safety of RCA versus no-anticoagulation in hy- 
pernatremia patients. During the past 2 decades, $>10$ randomized controlled trials have evaluated the efficacy of RCA versus heparin for continuous renal replacement therapy in patients without RCA and heparin contraindications, which mainly included liver failure and shock with muscle hypoperfusion (hyperlactacidemia) [46-48]. And some studies evaluate the efficacy and safety of citrate anticoagulation in patients with liver failure $[36,47]$ and hyperlactacidemia $[49,50]$. As aforementioned, the incidence of acute severe hypernatremia was significantly lower than liver failure and hyperlactacidemia. Only a few studies reported the use of $\mathrm{CVVH}$ for the treatment of acute severe hypernatremia patients, and none of these studies have evaluated the anticoagulation strategies for CVVH in these patients [18, 21-23]. Therefore, our present study firstly provided clinicians useful information in the conditions of using CVVH in acute severe hypernatremia patients with increased bleeding risk. Furthermore, to reduce the influence of the imbalance baseline characteristics on the results, we verified our findings by rigorous matching of the 2 groups with 3 variables related to the patient severity. This could be considered as another strength of our present study.

The retrospective nature should be considered as the major limitation of our present study. In a retrospective cohort study, not all of the desired laboratory data could be available at all time points of every patient. For example, the data of the patient coagulation indicators were not available for $32.8 \%$ of our patients. As we know, the coagulation indicators were commonly not influenced by using no-anticoagulation or RCA during CVVH treatment $[51,52]$. Therefore, these parameters were not tested after CVVH in some patients. However, the parameters of major outcomes were routinely examined in our daily work, and the observation time was covered by the duration of the patient's hospital stay. These characteristics could reduce the bias caused by missing data. Furthermore, this was a small single-center study. The results of a single-center study with small sample size are potentially easier to be associated with more systematic and selection bias. Further prospective controlled multi-center studies with large sample size are needed to validate our findings.

\section{Conclusions}

For acute severe hypernatremia patients with increased bleeding risk, RCA CVVH had a similar RRSe$\mathrm{Na}$ and longer filter survival time, compared with no- anticoagulation CVVH. Additionally, no serious adverse event of RCA was observed during the CVVH treatment. Most likely, RCA is a better anticoagulation method for CVVH than no-anticoagulation in acute severe hypernatremia patients with increased bleeding risk. Further prospectively designed studies with large sample size are warranted to provide stronger evidences on this field.

\section{Acknowledgements}

Not applicable.

\section{Statement of Ethics}

This retrospective study was approved by the Ethics Committee of Xijing Hospital and performed in accordance with the Declaration of Helsinki and waived the need for informed consent because of the retrospective study design.

\section{Disclosure Statement}

The authors have no potential conflicts of interest to disclose.

\section{Funding Sources}

This work was supported by the National Natural Science Foundation of China (81700584).

\section{Author Contributions}

M.B., S.S., L.Z., F.M., Y.Y., and Y.L. designed the study, analyzed the data, and drafted the manuscript. F.M. and Y.Y. collected and entered data. Y.W., L.H., M.Z., X.T., R.J., L.L., L.L., and C.H. contributed to the data acquisition and interpretation. All authors read and approved the final manuscript.

\section{Availability of Data and Materials}

The datasets used and/or analyzed during the current study are available from the corresponding author upon request.

\section{Consent for Publication}

Not applicable.
Zhao/Ma/Yu/Li/Wang/He/Zhou/Tian/ Jing/Li/Li/Huang/Bai/Sun 


\section{References}

1 Darmon M, Timsit JF, Francais A, NguileMakao M, Adrie C, Cohen Y, et al. Association between hypernatraemia acquired in the ICU and mortality: a cohort study. Nephrol Dial Transplant. 2010;25(8):2510-5.

2 Funk GC, Lindner G, Druml W, Metnitz B, Schwarz C, Bauer P, et al. Incidence and prognosis of dysnatremias present on ICU admission. Intensive Care Med. 2010;36(2):304-11.

3 Hoorn EJ, Betjes MG, Weigel J, Zietse R. Hypernatraemia in critically ill patients: too little water and too much salt. Nephrol Dial Transplant. 2008;23(5):1562-8.

4 Lindner G, Funk GC, Schwarz C, Kneidinger N, Kaider A, Schneeweiss B, et al. Hypernatremia in the critically ill is an independent risk factor for mortality. Am J Kidney Dis. 2007; 50(6):952-7.

5 Stelfox HT, Ahmed SB, Khandwala F, Zygun D, Shahpori R, Laupland K. The epidemiology of intensive care unit-acquired hyponatraemia and hypernatraemia in medical-surgical intensive care units. Crit Care. 2008; 12(6):R162.

6 Waite MD, Fuhrman SA, Badawi O, Zuckerman IH, Franey CS. Intensive care unit-acquired hypernatremia is an independent predictor of increased mortality and length of stay. J Crit Care. 2013;28(4):405-12.

7 Stelfox HT, Ahmed SB, Zygun D, Khandwala F, Laupland K. Characterization of intensive care unit acquired hyponatremia and hypernatremia following cardiac surgery. Can J Anaesth. 2010 Jul;57(7):650-8.

8 Lam NN, Minh NT. Risk factors and outcome of Hypernatremia amongst severe adult burn patients. Ann Burns Fire Disasters. 2018;31: 271-7.

9 Vedantam A, Robertson CS, Gopinath SP. Morbidity and mortality associated with hypernatremia in patients with severe traumatic brain injury. Neurosurg Focus. 2017;43(5):E2.

10 Lansink AO, Fahrentholz S, Nijsten MW. Risk of severe hypernatremia depends on underlying cause in critically ill patients. J Crit Care. 2013;28(2):213.

11 Kumar S, Berl T. Sodium. Lancet. 1998; 352(9123):220-8.

12 Palevsky PM. Hypernatremia. Semin Nephrol. 1998;18:20-30.

13 Bataille S, Baralla C, Torro D, Buffat C, Berland Y, Alazia M, et al. Undercorrection of hypernatremia is frequent and associated with mortality. BMC Nephrol. 2014;15(1):37.

14 Darmon M, Pichon M, Schwebel C, Ruckly $\mathrm{S}$, Adrie $\mathrm{C}$, Haouache $\mathrm{H}$, et al. Influence of early dysnatremia correction on survival of critically ill patients. Shock. 2014;41(5): 394-9.

15 Adrogué HJ, Madias NE. Hypernatremia. N Engl J Med. 2000;342(20):1493-9.

16 Alshayeb HM, Showkat A, Babar F, Mangold $\mathrm{T}$, Wall BM. Severe hypernatremia correction rate and mortality in hospitalized patients. Am J Med Sci. 2011;341(5):356-60.
17 O’Donoghue SD, Dulhunty JM, Bandeshe HK, Senthuran S, Gowardman JR. Acquired hypernatraemia is an independent predictor of mortality in critically ill patients. Anaesthesia. 2009;64(5):514-20.

18 Yang CW, Kim YS, Park IS, Chang YS, Yoon YS, Bang BK. Treatment of severe acute hypernatremia and renal failure by hemodialysis. Nephron. 1995;70(3):372-3.

19 Lin JJ, McKenney DW, Price C, Morrison RR, Novotny WE. Continuous venovenous hemodiafiltration in hypernatremic hyperglycemic nonketotic coma. Pediatr Nephrol. 2002; 17(11):969-73.

20 Yang YF, Wu VC, Huang CC. Successful management of extreme hypernatraemia by haemofiltration in a patient with severe metabolic acidosis and renal failure. Nephrol Dial Transplant. 2005;20(9):2013-4.

21 Huang C, Zhang P, Du R, Li Y, Yu Y, Zhou M, et al. Treatment of acute hypernatremia in severely burned patients using continuous veno-venous hemofiltration with gradient sodium replacement fluid: a report of nine cases. Intensive Care Med. 2013;39(8):1495-6.

22 Ostermann M, Dickie H, Tovey L, Treacher D. Management of sodium disorders during continuous haemofiltration. Crit Care. 2010; 14(3):418

23 Ma F, Bai M, Li Y, Yu Y, Liu Y, Zhou M, et al. Continuous Venovenous Hemofiltration (CVVH) Versus Conventional Treatment for Acute Severe Hypernatremia in Critically Ill Patients: A Retrospective Study. Shock. 2015; 44(5):445-51.

24 Sterns RH. Disorders of plasma sodiumcauses, consequences, and correction. $\mathrm{N}$ Engl J Med. 2015;372(1):55-65.

25 Ma F, Liu Y, Bai M, Li Y, Yu Y, Zhou M, et al. The Reduction Rate of Serum Sodium and Mortality in Patients Undergoing Continuous Venovenous Hemofiltration for Acute Severe Hypernatremia. Am J Med Sci. 2016; 352(3):272-9.

26 Fernandez SN, Santiago MJ, Lopez-Herce J, Garcia M, Del Castillo J, Alcaraz AJ, et al. Citrate anticoagulation for CRRT in children: comparison with heparin. BioMed Res Int. 2014;2014:786301.

27 Wu MY, Hsu YH, Bai CH, Lin YF, Wu CH, Tam $\mathrm{KW}$. Regional citrate versus heparin anticoagulation for continuous renal replacement therapy: a meta-analysis of randomized controlled trials. Am J Kidney Dis. 2012;59(6):810-8.

28 Ostermann M, Dickie H, Tovey L, Treacher D. Heparin algorithm for anticoagulation during continuous renal replacement therapy. Crit Care. 2010;14(3):419.

29 Palevsky PM, Liu KD, Brophy PD, Chawla LS, Parikh CR, Thakar CV, et al. KDOQI US commentary on the 2012 KDIGO clinical practice guideline for acute kidney injury. Am J Kidney Dis. 2013;61(5):649-72.

30 Bai M, Zhou M, He L, Ma F, Li Y, Yu Y, et al. Citrate versus heparin anticoagulation for continuous renal replacement therapy: an updated meta-analysis of RCTs. Intensive Care Med. 2015;41(12):2098-110.

31 Chen XM. Blood Purification Standard Operating Precedure(SOP). People's Army Medical Publishing House. China: Beijing; 2010. pp. 43-9.

32 Chua HR, Baldwin I, Bailey M, Subramaniam A, Bellomo R: Circuit lifespan during continuous renal replacement therapy for combined liver and kidney failure. J Crit Care. 2012 Dec; 27(6):744.e7-15.

33 Nurmohamed SA, Vervloet MG, Girbes AR Ter Wee PM, Groeneveld AB. Continuous venovenous hemofiltration with or without predilution regional citrate anticoagulation: a prospective study. Blood Purif. 2007;25(4): 316-23.

34 Lindner G, Funk GC: Hypernatremia in critically ill patients. J Crit Care. 2013 Apr;28(2): 216.e11-20.

35 Pinnick RV, Wiegmann TB, Diederich DA. Regional citrate anticoagulation for hemodialysis in the patient at high risk for bleeding. N Engl J Med. 1983;308(5):258-61.

36 Klingele M, Stadler T, Fliser D, Speer T, Groesdonk HV, Raddatz A. Long-term continuous renal replacement therapy and anticoagulation with citrate in critically ill patients with severe liver dysfunction. Crit Care. 2017;21(1):294

37 Kutsogiannis DJ, Gibney RT, Stollery D, Gao J. Regional citrate versus systemic heparin anticoagulation for continuous renal replacement in critically ill patients. Kidney Int. 2005;67(6):2361-7.

38 Oudemans-van Straaten HM. Citrate anticoagulation for continuous renal replacement therapy in the critically ill. Blood Purif. 2010; 29(2):191-6.

39 Monchi M, Berghmans D, Ledoux D, Canivet JL, Dubois B, Damas P. Citrate vs. heparin for anticoagulation in continuous venovenous hemofiltration: a prospective randomized study. Intensive Care Med. 2004;30(2): 260-5.

40 Oudemans-van Straaten HM, Bosman RJ, Koopmans M, van der Voort PH, Wester JP, van der Spoel JI, et al. Citrate anticoagulation for continuous venovenous hemofiltration. Crit Care Med. 2009;37(2):545-52.

41 Betjes MG, van Oosterom D, van Agteren M, van de Wetering J. Regional citrate versus heparin anticoagulation during venovenous hemofiltration in patients at low risk for bleeding: similar hemofilter survival but significantly less bleeding. J Nephrol. 2007;20: 602-8.

42 Morabito S, Pistolesi V, Tritapepe L, Zeppilli L, Polistena F, Strampelli E, et al. Regional citrate anticoagulation in cardiac surgery patients at high risk of bleeding: a continuous veno-venous hemofiltration protocol with a low concentration citrate solution. Crit Care. 2012;16(3):R111. 
43 Rewa OG, Eurich DT, Noel Gibney RT, Bagshaw SM. A modified Delphi process to identify, rank and prioritize quality indicators for continuous renal replacement therapy (CRRT) care in critically ill patients. J Crit Care. 2018;47:145-52.

44 Oudemans-van Straaten HM, Kellum JA, Bellomo R. Clinical review: anticoagulation for continuous renal replacement therapy-heparin or citrate? Crit Care. 2011;15(1):202.

45 Tolwani A, Wille KM. Advances in continuous renal replacement therapy: citrate anticoagulation update. Blood Purif. 2012;34(2):88-93.

46 Stucker F, Ponte B, Tataw J, Martin PY, Wozniak $\mathrm{H}$, Pugin J, et al. Efficacy and safety of citrate-based anticoagulation compared to heparin in patients with acute kidney injury requiring continuous renal replacement therapy: a randomized controlled trial. Crit Care. 2015;19(1):91.
47 Zhang W, Bai M, Yu Y, Li L, Zhao L, Sun S, et al. Safety and efficacy of regional citrate anticoagulation for continuous renal replacement therapy in liver failure patients: a systematic review and meta-analysis. Crit Care. 2019; 23(1):22.

48 Schilder L, Nurmohamed SA, Bosch FH, Purmer IM, den Boer SS, Kleppe CG, et al. group Cs: Citrate anticoagulation versus systemic heparinisation in continuous venovenous hemofiltration in critically ill patients with acute kidney injury: a multi-center randomized clinical trial. Crit Care. 2014;18(4): 472.

49 Khadzhynov D, Dahlinger A, Schelter C, Peters H, Kindgen-Milles D, Budde K, et al. Hyperlactatemia, Lactate Kinetics and Prediction of Citrate Accumulation in Critically Ill Patients Undergoing Continuous Renal Replacement Therapy With Regional Citrate
Anticoagulation. Crit Care Med. 2017;45(9): e941-6.

50 Tan JN, Haroon SW, Mukhopadhyay A, Lau T, Murali TM, Phua J, et al. Hyperlactatemia Predicts Citrate Intolerance With Regional Citrate Anticoagulation During Continuous Renal Replacement Therapy. J Intensive Care Med. 2019;34(5):418-25.

51 Tuerdi B, Zuo L, Sun H, Wang K, Wang Z, Li G. Safety and efficacy of regional citrate anticoagulation in continuous blood purification treatment of patients with multiple organ dysfunction syndrome. Braz J Med Biol Res. 2017 Nov 17;51(1):e637.

52 Gille J, Sablotzki A, Malcharek M, Raff T, Mogk M, Parentin T: Regional citrate anticoagulation for continuous renal replacement therapy in severe burns-a retrospective analysis of a protocol-guided approach. Burns. 2014 Dec;40(8):1593-601. 